\title{
Acepciones dialectales en el léxico de la ganadería en el departamento de Casanare, Colombia*
}

\author{
MARÍA ISABEL VACA VEGA** \\ Institución Educativa Jorge Eliécer Gaitán del \\ Municipio de Yopal, Colombia. \\ mariaisabelvacana@hotmail.com \\ DELFÍN RIVERA SALCEDO \\ Poeta, historiador, escritor y periodista \\ en el Departamento de Casanare, Colombia \\ delfinhuesito@hotmail.com
}

Recepción: 17 de agosto de 2016

Aprobación: 27 de septiembre de 2016

Forma de citar este artículo: Vaca Vega, M.I., \& Rivera Salcedo, D. (2017). Acepciones dialectales en el léxico de la ganadería en el departamento de Casanare, Colombia. Cuadernos de Lingüística Hispánica, (29), 63-82. doi: http://dx.doi.org/10.19053/0121053X. n29.2017.5847

* Artículo resultado de la investigación "Léxico de la ganadería en Casanare".

** Candidata a Magíster en Lingüística. Especialista en Edumática de la Universidad Autónoma de Colombia. Licenciada en Educación Básica Primaria, de la Pontificia Universidad Javeriana. Docente en la Institución Educativa Jorge Eliécer Gaitán del Municipio de Yopal, Casanare. Código ORCID: orcid.org/0000-0001-7838-7848

** Candidato a Magíster en Lingüística. Poeta, historiador y escritor con diecisiete obras publicadas. Especialista en Alta Gerencia de la Corporación Universitaria del Meta, Unimeta; Especialista en Dirección y Gestión Deportiva de la Escuela Nacional del Deporte en Cali. Comunicador Social Periodista de la Fundación Universidad de Bogotá Jorge Tadeo Lozano. Código ORCID: orcid.org/00000002-0403-4473 


\section{Resumen}

Este artículo hace parte del proyecto de investigación "Léxico dialectal de la ganadería en Casanare, Colombia", que tiene como propósitos caracterizar esa comunidad de habla y mostrar los fenómenos lingüísticos usados, su léxico autóctono y su impacto sociológico en esta colectividad llanera. La presente investigación es un estudio dialectal de tipo descriptivoexplicativo con tendencia cuantitativa-cualitativa; aquí se describe de manera teórica y empírica la predisposición del uso de expresiones dialectales. Una vez realizado el análisis, se encontró que el uso de los términos dialectales de la ganadería en Casanare (Colombia) se convirtió en un recurso retórico, que cumple funciones pragmáticas de acercamiento del emisor a sus interlocutores; asimismo, que las variantes, la sinonimia y la polisemia son una característica común a muchas de las piezas léxicas que presentan varias alternativas para referirse a lo mismo.

Palabras clave: léxico, dialecto, ganadería, Casanare.

\section{Dialectal Meanings in the Livestock Farming Lexicon of the Region of Casanare, Colombia}

\section{Abstract}

This article is part of the research project Dialectal Lexicon from Livestock Farming Community in Casanare, Colombia. This project seeks to characterize this speech community and identify some of its usual linguistic phenomena, autochthonous vocabulary and sociological impact in this area. The research was a descriptive-explanatory dialectal study with a quantitative-qualitative tendency, which theoretically and empirically describes the the use of certain dialectal expressions. Through this analysis, it was found that, dialectal terms in livestock farming community from Casanare (Colombia) have become a rhetorical resource with a pragmatic role: enabling an easier and more fluid contact between conversational partners. In the same way, we found that linguistic variations, synonymy and polysemy are usual characteristics of most of the lexical samples studied, which present different alternatives to refer to the same things.

Key words: Vocabulary, Dialect, Livestock Farming, Casanare. 


\section{Acceptions dialectales dans le lexique de l'élevage de bétail dans le département de Casanare, Colombie}

\section{Résumé}

Cet article fait partie du projet de recherche "Lexique dialectal de l'élevage du bétail à Casanare, Colombie", ayant comme les buts de caractérise cette communauté-là de parole et montrer les phénomènes linguistique utilisés, leur lexique autochtone et leur impact sociologique dans cette communauté de la plaine colombienne. Cette recherche est une étude dialectale de type descriptif-explicatif ayant une tendance quantitative-qualitative; on décrit ici, de manière théorie et empirique, la prédisposition de usage des expressions dialectales. Une fois l'étude faite, on a trouvé que l'utilisation des termes dialectaux de l'élevage de bétail à Casanare (Colombie) est devenue une source théorique qui remplit des fonctions pragmatiques de rapprochement de l'émetteur à leurs interlocuteurs. De la même manière que les variantes, la synonymie et la polysémie constituent une caractéristique commune à plusieurs des pièces lexicales qui présentent diverses alternatives pour faire référence à soi-même.

Mots clés: lexique, dialecte, élevage de bétail, Casanare.

\section{Acepções dialectais no léxico da pecuária no departamento de Casanare, Colômbia}

\section{Resumo}

Este artigo faz parte do projeto de pesquisa "Léxico dialectal da pecuária em Casanare, Colômbia", que tem como propósitos caracterizar essa comunidade na maneira de falar e mostrar os fenômenos linguísticos usados, seu léxico autóctone e seu impacto sociológico nesta coletividade llanera (Llano: uma região da Colômbia). A presente pesquisa é um estudo dialectal de tipo descritivo-explicativo com tendência quantitativa-qualitativa; aqui descreve-se de maneira teórica e empírica a predisposição do uso de expressões dialectais. Uma vez realizada a análise, encontrou-se que 0 uso dos termos dialectais da pecuária em Casanare (Colômbia) converteu-se em um recurso retórico, que cumpre funções pragmáticas de aproximação do emissor a seus interlocutores; da mesma forma que as variantes, a sinonímia e a polissemia são uma característica comum a muitas das peças léxicas que apresentam várias alternativas para dizer-se a mesma coisa.

Palavras chave: léxico, dialeto, pecuária, Casanare. 


\section{Introducción}

Desde tiempos muy remotos, la dialectología ha sido una de las manifestaciones lingüísticas que permite el conocimiento de la realidad lingüística a partir de una recogida del léxico. Por lo tanto, conocer bien una lengua supone conocer de sus distintas manifestaciones lingüísticas, entre ellas, el léxico principal representante de la lengua popular. De ahí que, una lengua no se pueda comprender sin la cultura en la que se manifiesta. El léxico lleva implícita la sabiduría, las costumbres, la historia de la sociedad (Sevilla y Barbadillo, 2007). La vida cultural de los pueblos muestra una forma particular de organizar y de percibir el mundo. La lengua es uno de los recursos del hombre para expresarlo. El léxico propio de una región refleja los recursos, los oficios, la gastronomía, el trabajo, la manera de cultivar la tierra y de nombrar todo el contexto.

Por lo anterior, este estudio tiene como referente al llanero casanareño, quien ha llevado hasta ahora un género de vida pastoril, en el que los grandes espacios abiertos, el manejo de manadas de rumiantes de los que deriva su sustento, y el enderezamiento de todas las actividades vivenciales hacia dicha actividad, son sus principales características. De tal forma que, en tales condiciones, cobra desmedida devoción a sus expresiones y hacia ellas orienta sus contenidos vivenciales; es decir todo lo referencia con el llano y su vida ganadera. Por lo tanto, si se afectara la producción ganadera y su hábitat, se modificarían sus hábitos culturales, su lenguaje y hasta su sensibilidad. Entonces, la vida del llanero casanareño reúne un léxico rico: los animales y sus cuidadores, las plantas de la sabana, los males que aquejan a las bestias y el bastimento que acompaña al vaquero en su diario vivir, en sus largas y esforzadas jornadas.

La competencia léxica da al hablante la posibilidad de usar un sistema lingüístico adecuado y apropiado; y, al enriquecer su léxico, lo dota de la acepción exacta en cada situación comunicacional. El aprendizaje del léxico es una constante en las personas. Las estructuras gramaticales, sintácticas, morfológicas, etc., aprehendidas en la lengua materna quedan fijadas y son estables; el léxico no es estático, sino que es dialéctico, esto es, que está en permanente cambio y su aprendizaje termina con la desaparición del ser humano. Por lo tanto, este trabajo de investigación recoge el léxico dialectal que usa la población ganadera, y que constituye el sentido de identidad llanera en la región del departamento de Casanare, toda vez que este ha venido desapareciendo en forma alarmante y, por tanto, ha dejado de ser un instrumento de comunicación entre los llaneros casanareños. 
El español es la lengua oficial hablada en Casanare junto con un sinnúmero de lenguas indígenas 4 . En la conformación del español casanareño, además del castellano andaluz, encontramos elementos lingüísticos, indígenas y africanos. El diverso origen dialectal de los colonizadores, la diversidad de lenguas aborígenes, el aislamiento de los núcleos fundacionales, la ausencia de políticas lingüísticas niveladoras permitió el surgimiento de un dialecto propio de la actividad ganadera en Casanare, que identifica a los llaneros casanareños (Rivera, 2001).

Hablar de la identidad cultural llanera es hablar de los grupos de poblaciones asentados en los llanos colombianos, indígenas, llaneros. Sin embargo, existen otros grupos que han provocado los cambios culturales en los pobladores con quienes han tenido contacto. Estos grupos son: narcos, paramilitares y guerrilleros. Se puede clasificar a los tres últimos como grupos móviles que aparecen y desaparecen en la región, según los vaivenes de sus actividades ilícitas, pero que van dejando su idiolecto, sus costumbres y sus creencias. Los llaneros y los indígenas son grupos fijos y tienen terrenos establecidos de movilidad, con un espacio definido, que forma parte importante de su identidad. Dentro del grupo de los llaneros y de los tres llamados móviles, existe una diferenciación interna dada por el contraste campo-ciudad, que señala la frontera entre lo tradicional y lo conservador frente a lo moderno y evolucionado.

La globalización, las migraciones poblacionales, las relaciones comerciales, las nuevas formas de producción, el desarrollo de la tecnología y el impacto de los medios de comunicación han traído consigo cambios terminológicos (nuevos vocablos, expresiones) que se arraigan en los hablantes casanareños y desplazan el vocabulario regional hasta llevarlo a la desaparición. En consecuencia, la riqueza léxica que, por centurias, usaron los llaneros ganaderos del Casanare y que mantuvo vivas las relaciones interindividuales están en inminente peligro de extinción.

En la actualidad, las investigaciones lexicográficas sobre el vocabulario de uso llanero-ganadero en ciudades, municipios, corregimientos y veredas del departamento de Casanare no existen, lo que origina carencia de información cuando se intenta establecer contrastes 0 visiones acerca de las relaciones lingüísticas y sociales que determinan la dinámica de los procesos comunicativos. Por consiguiente, este trabajo de investigación tuvo como objetivo, caracterizar el léxico dialectal llanero de la ganadería en Casanare para elaborar un diccionario de usos; y como objetivos específicos, recoger dicho léxico-dialectal,

4 Las etnias indígenas que existen en la actualidad en Casanare son: Achaguas, Amorua, Cuiba, Maiben-Masiware, Sálivas, Sikuani, Tsiripo, W'a, Wipiwi, Yamalero, Yaruro. 
sistematizar por campos semánticos la información obtenida, analizarla, caracterizarla y elaborar un diccionario de uso dialectal.

Si el problema de esta investigación, fue ante todo ¿cómo caracterizar el léxico dialectal de la ganadería en Casanare y elaborar un diccionario de uso de esta actividad?, entonces, este estudio se justificó en la medida en que respondió a la necesidad de indagar sobre la realidad lingüística casanareña que no se había abordado. Por lo tanto, se ubica en el campo de la dialectología y la lexicografía, porque emplea unidades léxicas dialectales que expresan los hablantes ganaderos del departamento de Casanare.

Así pues, la realización de este estudio permitió recoger y caracterizar el léxicodialectal de la ganadería en Casanare -en su estado actual- y compendiarlo en un diccionario, para evitar su desaparición y así contribuir con el afianzamiento de la identidad llanera de los casanareños.

Entre los investigadores que han reclamado la conveniencia de tener en cuenta el ¿para qué?, ¿para quién? y icon qué finalidad? se recoge, se caracteriza y se elabora un corpus dialectal, se encuentran Fajardo (1993) y, en particular, Werner (1993-1994) y en cuanto a la elaboración de diccionarios, la lexicografía actual reitera la necesidad de determinar a quién va dirigido y cuál es su finalidad, ya que de los fines y los destinatarios previstos dependerán tanto la selección de los elementos léxicos que formarán la macroestructura del diccionario, como también la información que se proporcione sobre cada uno de ellos. Es importante tener en cuenta los parámetros aludidos, puesto que todo léxico recogido, caracterizado y plasmado en un diccionario es una obra de consulta, cuya naturaleza didáctica resulta, por tanto, indiscutible (Alvar, 1996; Hernández, 1998; Porto, 2002). Estos planteamientos son igualmente válidos cuando se trata de elaborar repertorios lexicográficos que registren unidades léxicas diatópicamente restringidas, esto es, diccionarios dialectales, también llamados regionales, de dialectalismos, de regionalismos o, en los últimos años, diferenciales.

Este trabajo está dirigido a los pobladores de Casanare, a las instituciones pedagógicas, a las academias y a los investigadores de la lengua castellana como un aporte de la variedad lingüística regional casanareña. De igual manera, contribuirá a la conservación del patrimonio lingüístico del habla ganadera llanera en Casanare, como parte de la cultura inmaterial del departamento. Además, se constituirá en fuente de consulta para futuros investigadores y estudiantes tanto de las ciencias del lenguaje como de otras áreas del conocimiento. 


\section{Diseño metodológico}

La presente investigación es un estudio dialectal de tipo descriptivo-explicativo con tendencia cuantitativa-cualitativa. La investigación descriptiva trabaja sobre realidades de hecho y su caracterización fundamental es presentar una interpretación correcta de la naturaleza u objeto de estudio, que en el presente caso es El léxico dialectal llanero de la ganadería en Casanare, para elaborar un diccionario de usos. Danhke (citado por Sampieri et al., 2010) afirma que "los estudios descriptivos buscan especificar las propiedades, las características y los perfiles de personas, grupos, comunidades, procesos, objetos o cualquier otro fenómeno que se someta a análisis" (p. 102). Los estudios descriptivos miden, evalúan $o$ recolectan datos sobre diferentes variables o conceptos, dimensiones o componentes del fenómeno o hecho por investigar.

Para la presente investigación, se tendrán en cuenta los enfoques cuantitativo -que mide numéricamente los resultados-; y cualitativo -análisis de los datos presentados por los informantes para la interpretación correspondiente-. Como lo definen Sampieri et al. (2010), "el enfoque cuantitativo usa la recolección de datos para probar hipótesis, con base en la medición numérica y el análisis estadístico, para establecer patrones de comportamiento y probar teorías"; mientras que, "el enfoque cualitativo utiliza la recolección de datos sin medición numérica para descubrir 0 afinar preguntas de investigación en el proceso de interpretación" (pp. 5, 8).

Al observar la imposibilidad de describir los hechos sin explicarlos, se deduce que descripción y explicación están estrechamente ligadas y se transforman dialécticamente una con respecto a la otra; ante esta realidad, Sampieri et al. (2010) plantean que: "el interés de un estudio explicativo se centra en explicar por qué ocurre un fenómeno y en qué condiciones se manifiesta o por qué se relacionan dos o más variables" (p. 108).

La dialectología se enmarca dentro de la investigación de tipo descriptivo, ya que permite describir el aspecto de una lengua, en este caso: el léxico dialectal en un territorio, el departamento de Casanare, dentro de un contexto que hace referencia a las actitudes y comportamientos sociales y lingüísticos de sus habitantes.

La dialectología, según Montes (1995) corresponde a la intraidiomática o estudio de la articulación de las normas dentro de un sistema lingüístico-normativo autónomo (= lengua histórica). Es decir, la función de la dialectología es el estudio de la unidad en la variedad, lo que equivale a expresar la forma en que un conjunto de normas, variedades y variantes se integra en un conjunto mayor (Heilmann, 1964). 
El método descriptivo, en esta investigación, permitió seleccionar las características fundamentales del léxico dialectal de la ganadería en Casanare y su descripción detallada dentro del marco de referencia, ubicando los indicadores cuantitativos y cualitativos con el fin de hacer más viable y factible el objetivo de estudio -centrado en el léxico dialectal ganadero en Casanare- (Hernández, 2008).

\section{Población}

La población objeto de estudio se conformó con los habitantes del sector rural del departamento de Casanare, Colombia, que se desempeñan en la actividad ganadera. Personas que han nacido en este departamento y otras que por diversos motivos se encuentran actualmente radicadas en Casanare. Según el último censo realizado en Colombia (Dane, 2005), Casanare contaba aproximadamente con 281.294 habitantes, de los cuales 196.508 viven en las zonas urbanas y 84.786 viven en la zona rural.

En el departamento del Casanare, el ganado bovino ocupa el renglón más importante dentro de la actividad pecuaria, gracias a los ingresos que genera a nivel municipal como departamental, excluyendo el petróleo.

\section{Muestra}

La muestra que se tomó tiene un índice de 1/25.000, y corresponde al estándar propuesto por el proyecto PRESEEA 5 , con base en los parámetros establecidos por este proyecto; es decir, alrededor de 2.000 .000 de habitantes. Ahora bien, en los núcleos de habitantes inferiores a 500.000 (como es el caso del departamento de Casanare), es posible reducir a tres el número de informantes por cuota, lo que daría una muestra de 54 informantes (1/9.250), es decir que la muestra seleccionada para este trabajo estaría por encima de esos lineamientos, puesto que Casanare cuenta con una población de 281.294 habitantes, de los cuales 196.508 viven en las zonas urbanas y 84.786 están en la zona rural. Según estos datos, la muestra poblacional se constituyó con 90 personas ( 70 hombres y 20 mujeres), 10 en cada municipio, nacidos en el departamento de Casanare o que llevan viviendo allí más de treinta años y son representativos en el oficio de la ganadería ${ }^{6}$. En esa población se aplicaron 90 encuestas directas.

Los más recientes avances teóricos y metodológicos en geografía lingüística evidencian una innovación crucial en conceptos de uso común, como espacio, región y

5 Véase el siguiente enlace: http://www.linguas.net/preseea.

6 Aquí se entiende la ganadería como la ganadería vacuna y equina. 
territorio, que por su transformación metodológica y conceptual tienen un impacto en los estudios del lenguaje. Es así, como:

[... La dialectología tradicional toma en cuenta la variación existente entre regiones [...] En los estudios lingüísticos se ha presentado un cambio muy importante, la dialectología ya no es la misma del pasado porque la forma de espacializar fenómenos relacionados con la lengua son distintas; ahora el trabajo geográfico puede ser complementado y registrado con el uso cuantitativo y estadístico, los sistemas de posicionamiento global (GPS), motores de bases de datos especiales y sistemas de información geográfica (SIG). Avances asociados a la adopción de tecnologías, cartografías técnicas y métodos cuantitativos que ya venían apoyando la investigación de la lengua en relación con la geografía. (Reyes y Córdoba, 2009, p. 146).

\section{Prueba piloto}

Para el efecto de la viabilidad y confiabilidad del cuestionario, se aplicó una prueba piloto a 9 personas de los municipios, donde se llevó a cabo la encuesta (una por cada municipio), con el fin de comprobar la validez del instrumento que posteriormente se modificó para su aplicación en el terreno.

\section{Instrumentos para la recolección de la muestra}

Tabla 1. Datos de las fincas y de los informantes seleccionados para la prueba piloto

\begin{tabular}{l|l|c|c|c|c|c|c}
\hline $\begin{array}{c}\text { Nombre } \\
\text { de la finca, } \\
\text { fundo o hato }\end{array}$ & $\begin{array}{l}\text { Nombre del propietario } \\
\text { o administrador }\end{array}$ & $\begin{array}{c}\text { Sexo } \\
\mathrm{m}\end{array}$ & $\begin{array}{c}\text { Sexo } \\
\mathrm{f}\end{array}$ & $\begin{array}{c}\text { Tiempo que lleva } \\
\text { viviendo en la finca }\end{array}$ & $\begin{array}{c}\text { Lugar de } \\
\text { nacimiento }\end{array}$ & $\begin{array}{c}\text { Tiempo } \\
\text { trabajando } \\
\text { en la zona }\end{array}$ & $\begin{array}{c}\text { Nivel de } \\
\text { instrucción }\end{array}$ \\
\hline $\begin{array}{l}\text { La } \\
\text { Cerdeñosa }\end{array}$ & $\begin{array}{l}\text { Hugoberto Huertas } \\
\text { Ramírez }\end{array}$ & $\mathrm{X}$ & 49 & Villanueva & 50 & Pregrado \\
\hline La Frontera & $\begin{array}{l}\text { Gustavo Eustasio } \\
\text { Barrera }\end{array}$ & $\mathrm{X}$ & 70 & Yopal & 70 & Primaria \\
\hline La Colonia & $\begin{array}{l}\text { Francisco Alberto } \\
\text { Sandoval }\end{array}$ & $\mathrm{X}$ & 40 & Tauramena & 40 & Posgrado \\
\hline La Laguna & Armando Betancourt & $\mathrm{X}$ & 40 & Maní & 45 & Primaria \\
\hline $\begin{array}{l}\text { La } \\
\text { Candelaria }\end{array}$ & Tadeo Márquez & $\mathrm{X}$ & 57 & Orocué & 60 & Primaria \\
\hline $\begin{array}{l}\text { Vida } \\
\text { Tranquila }\end{array}$ & Orlando Valderrama & $\mathrm{X}$ & & 45 & Pore & 50 & Secundaria \\
\hline E1 Nido & $\begin{array}{l}\text { Rebeca Salcedo } \\
\text { Rodríguez }\end{array}$ & & $\mathrm{X}$ & 50 & Trinidad & 50 & Primaria \\
\hline El Carmen & Cilia Zambrano & & $\mathrm{X}$ & 60 & Támara & 45 & Primaria \\
\hline El Poyero & José María Rivera & $\mathrm{X}$ & & 90 & Hatocorozal & 65 & Primaria \\
\hline
\end{tabular}


Como instrumento de recolección, se diseñó y aplicó una encuesta que permitió la recolección sistemática de los datos de la muestra. Dicha encuesta consta de un encabezado, referencias o datos personales del encuestado y 60 ítems correspondientes al objetivo trazado para la investigación.

La aplicación de la encuesta a la comunidad ganadera de Casanare, se realizó durante el segundo semestre de 2013; se respondieron 90 cuestionarios aplicados a 9 personas, en cada uno de los siguientes municipios: Trinidad, Orocué, Villanueva, Yopal, Hato Corozal, Pore, Nunchía, Maní, Támara y Tauramena, individuos nacidos en el departamento de Casanare o que llevan viviendo allí más de treinta años, y que son representativas del oficio de la ganadería.

Asimismo, se utilizó la entrevista semiestructurada que, como su nombre lo indica, "se caracteriza porque el entrevistador tiene trazado un plan de desarrollo, pero procede con más libertad de acción y mayor agilidad" (Ibáñez y López, citados en Calderón y Alvarado, 2011, p. 14). Se diligenciaron 10 entrevistas, una por cada municipio, con las cuales se construyeron testimonios, actitudes y creencias acerca de las acepciones dialectales encontradas en las encuestas.

Por ser la observación la compenetración del investigador en una variedad de actividades durante un extenso periodo de tiempo, que le permite observar a los miembros culturales en sus vidas diarias y participar en sus actividades para facilitar una mejor comprensión de esos comportamientos y actividades, se utilizó la observación directa como otra técnica de la investigación.

\section{Fases de la recolección y elaboración del léxico}

Para la recolección del léxico y la elaboración del diccionario, se siguió la propuesta dialectológica de Montes (1995). En una primera fase, se recolectó el léxico-dialectal de la ganadería en Casanare. Mediante la información oral, se conformó el corpus. Se realizaron 90 encuestas ( 9 en cada municipio), como ya se informó, a personas nacidas en el departamento de Casanare o que llevan viviendo allí más de treinta años y que son representativas en el oficio de la ganadería. Se tuvo en cuenta las variables: nombre de la finca, fundo o hato, nombre del propietario o administrador, sexo, tiempo que lleva viviendo en la zona, lugar de nacimiento, tiempo trabajando en la zona y nivel de instrucción, de la siguiente manera: 
Tabla 2. Datos de las fincas y de los informantes seleccionados

\begin{tabular}{cccccccc}
\hline $\begin{array}{c}\text { Nombre de la } \\
\text { finca, fundo o } \\
\text { hato }\end{array}$ & $\begin{array}{c}\text { Nombre del } \\
\text { propietario 0 } \\
\text { administrador }\end{array}$ & $\begin{array}{c}\text { Sexo } \\
\text { M }\end{array}$ & $\begin{array}{c}\text { Sexo } \\
\text { F }\end{array}$ & $\begin{array}{c}\text { Tiempo } \\
\text { que lleva } \\
\text { viviendo en } \\
\text { la zona }\end{array}$ & $\begin{array}{c}\text { Lugar de } \\
\text { nacimiento }\end{array}$ & $\begin{array}{c}\text { Tiempo } \\
\text { trabajando } \\
\text { en la zona }\end{array}$ & $\begin{array}{c}\text { Nivel de } \\
\text { instrucción }\end{array}$ \\
\hline Las Maracas & Carlos Ramos & $\mathrm{X}$ & & 79 & Nunchía & 70 & Primaria \\
La Cerdeñosa & $\begin{array}{c}\text { Hugoberto Huertas } \\
\text { El Carmen }\end{array}$ & $\mathrm{X}$ & & 60 & Villanueva & 50 & Pregrado \\
\hline
\end{tabular}

Se visitó a cada encuestado en su casa o en su lugar de trabajo; allí se comenzó una conversación informal dirigida a situaciones cotidianas y típicas de la vida ganadera en el departamento de Casanare. Los informantes respondieron a un cuestionario preestablecido con temas de usos de la ganadería. Los encuestados pertenecen al área rural y desempeñan algún oficio relacionado con la ganadería en el departamento de Casanare.

La encuesta se realizó con orientación onomasiológica, es decir, se construía una idea en forma de pregunta para que el informante respondiera con la palabra 0 el significante. Un total de 60 preguntas junto con sus respuestas se grabaron en audio y después se trascribieron.

En la segunda fase, se procedió a sistematizar por campos semánticos la información obtenida, analizarla, caracterizarla, categorizarla y finalmente, se elaboró un diccionario de usos.

\section{Análisis de la información}

Una vez recogido el léxico de la ganadería en Casanare, se procedió a la caracterización de los materiales, indagando en periódicos y obras escritas en la región, con el fin de comprobar la aparición de las unidades léxicas obtenidas en las encuestas. Los documentos consultados fueron: Atlas lingüístico-etnográfico de Colombia (Instituto Caro y Cuervo, 1981); Caracterización Léxica de los dialectos del español de Colombia según el Alec (Instituto Caro y Cuervo, 2004); Idiomas y etnografía de la Región Oriental de Colombia (Fabo, 1911); Del folclor llanero (Martín, 1979); Habla y cultura popular en los llanos de Casanare (Cáceres, Salamanca y Mariño, 1988).

Luego, se seleccionó el material y se elaboró un segundo listado de unidades léxicas con las posibles acepciones según su contexto de uso. Después, con el fin de verificar si aparecía registrada o no la unidad léxica y con qué acepción, se revisaron los siguientes diccionarios: Diccionario de la lengua española (2012); Diccionario de americanismos 
(2010); Breve diccionario de colombianismos (2012); Diccionario sikuani-español (Quixalós, 1988); Diccionario achagua-español/español-achagua (Meléndez Lozano, 2011).

Esta labor de verificación permitió diferenciar usos particulares de la región frente a otras acepciones de diversas zonas del país; además, esta sirvió como delimitación de las posibles entradas para el diccionario y, de esta manera, se determinó reducir el corpus. Con miras a cotejar la información recolectada y obtener el corpus final, se revisaron las encuestas y el Alec; también se tuvo en cuenta la diatopía registrada por Haensch et al. (2012). Esta etapa corroboró la existencia de las unidades léxicas-dialectales de la ganadería en el departamento de Casanare. Desarrollada esta labor se establecieron los parámetros para escoger las entradas definitivas: el registro en fuentes escritas de la región y la existencia en el Alec, el Drae, el Diccionario de americanismos, el Nuevo diccionario de colombianismos, el Diccionario achagua-español español-achagua y el Diccionario sikuani-español.

La redacción de las definiciones incluyó la información básica sobre las restricciones de uso de las unidades léxicas que aparecen en el diccionario, es decir, las marcas. En general, estas varían según el propósito del diccionario y sus usuarios; pueden referirse a la etimología, categoría gramatical, ortografía; además, señalan si esa unidad "tiene plena vigencia en la lengua, si se utiliza en una determinada área geográfica, si es propia de una determinada profesión o actividad, o si está restringida a un determinado nivel o registro lingüístico" (Medina, 2003, p. 107).

Las marcas se ubicaron con una abreviatura antes de la definición y se clasificaron según lo propone Medina (2003) en diacrónicas, diatópicas, diastráticas y diatécnicas. Las marcas diacrónicas indican la vigencia de uso de una palabra (anticuado, desusado, poco usado, obsoleto, arcaico). Las marcas diatópicas informan sobre las restricciones de tipo geográfico en el uso de una palabra. Las marcas diafásicas y diastráticas se refieren al estilo, el nivel de lengua y la intención (p. 108).

Otras marcas son las de transición semántica, en las que se intentó mostrar un cambio de significado según el lenguaje figurado (metafórico, metonímico) y, por último, la marca diatécnica, que hace referencia a una ciencia o disciplina en particular (lingüística, etc.).

Siguiendo a Medina (2003), en este léxico se tuvieron en cuenta las siguientes marcas: 
Marcas gramaticales: son aquellas referidas a la clasificación gramatical de las entradas del léxico.

$\begin{array}{lll}\text { Adjetivo: } & & \text { adj. } \\ \text { Adjetivo } & \text { sustantivo: } & \text { adj./s } \\ \text { Plural: } & & \mathrm{pl} . \\ \text { Sustantivo } & \text { femenino: } & \mathrm{f} . \\ \text { Sustantivo } & \text { masculino } & \mathrm{m} . \\ \text { Sustantivo } & \text { masculino o femenino: } & \mathrm{s} . \\ \text { Verbo } & \text { intransitivo: } & \text { intr. } \\ \text { Verbo } & \text { transitivo: } & \text { tr. }\end{array}$

Registros específicos: evidencian desde la diastratía, el uso particular de una capa o grupo social. En el léxico-dialectal de la ganadería en Casanare, se determinaron expresiones según la edad.

Marcas de transición semántica: los procesos más frecuentes de creación léxica son la metáfora y la metonimia, que tienen como finalidad modificar el significado de las palabras.

Metáfora: $\quad$ metaf.

Metonimia: meton.

En lo referente a la lematización y ordenación de las entradas, se siguieron criterios según la categoría gramatical a la cual pertenece la unidad léxica (sustantivos y adjetivos).

\subsection{Análisis cuantitativo}

El análisis cuantitativo tiene tres objetivos principales: primero, averiguar el grado de conocimiento del léxico usado en la ganadería de las 90 personas encuestadas; segundo, la identificación de la o las voces que pertenecen a la lengua estándar, para así definir el total de voces correspondientes al léxico dialectal de la ganadería; y, tercero, la cuantificación de voces encontradas en los diccionarios consultados. 
Tabla 3. Consolidado voces recogidas en los municipios encuestados

\begin{tabular}{lccc}
\hline \multicolumn{4}{c}{ CONSOLIDADO } \\
\hline \multicolumn{1}{c}{ MUNICIPIO } & $\begin{array}{c}\text { TOTAL DE VOCES } \\
\text { ENCONTRADAS EN } \\
\text { HOMBRES ENCUESTADOS }\end{array}$ & $\begin{array}{c}\text { TOTAL DE VOCES } \\
\text { ENCONTRADAS EN } \\
\text { MUJERES }\end{array}$ & $\begin{array}{c}\text { TOTAL ABSOLUTO } \\
\text { DE VOCES } \\
\text { ENCONTRADAS }\end{array}$ \\
\hline Hatocorozal & 7514 & 2122 & 9638 \\
Maní & 7554 & 2139 & 9691 \\
Nunchía & 7474 & 2122 & 9594 \\
Orocué & 7502 & 2120 & 9626 \\
Pore & 7483 & 2070 & 9542 \\
Tauramena & 7394 & 2075 & 9465 \\
Trinidad & 7570 & 2151 & 9716 \\
Villaneva & 7223 & 2034 & 9257 \\
Támara & 7034 & 2005 & 9031 \\
Yopal & 6968 & 1987 & 8955 \\
\hline
\end{tabular}

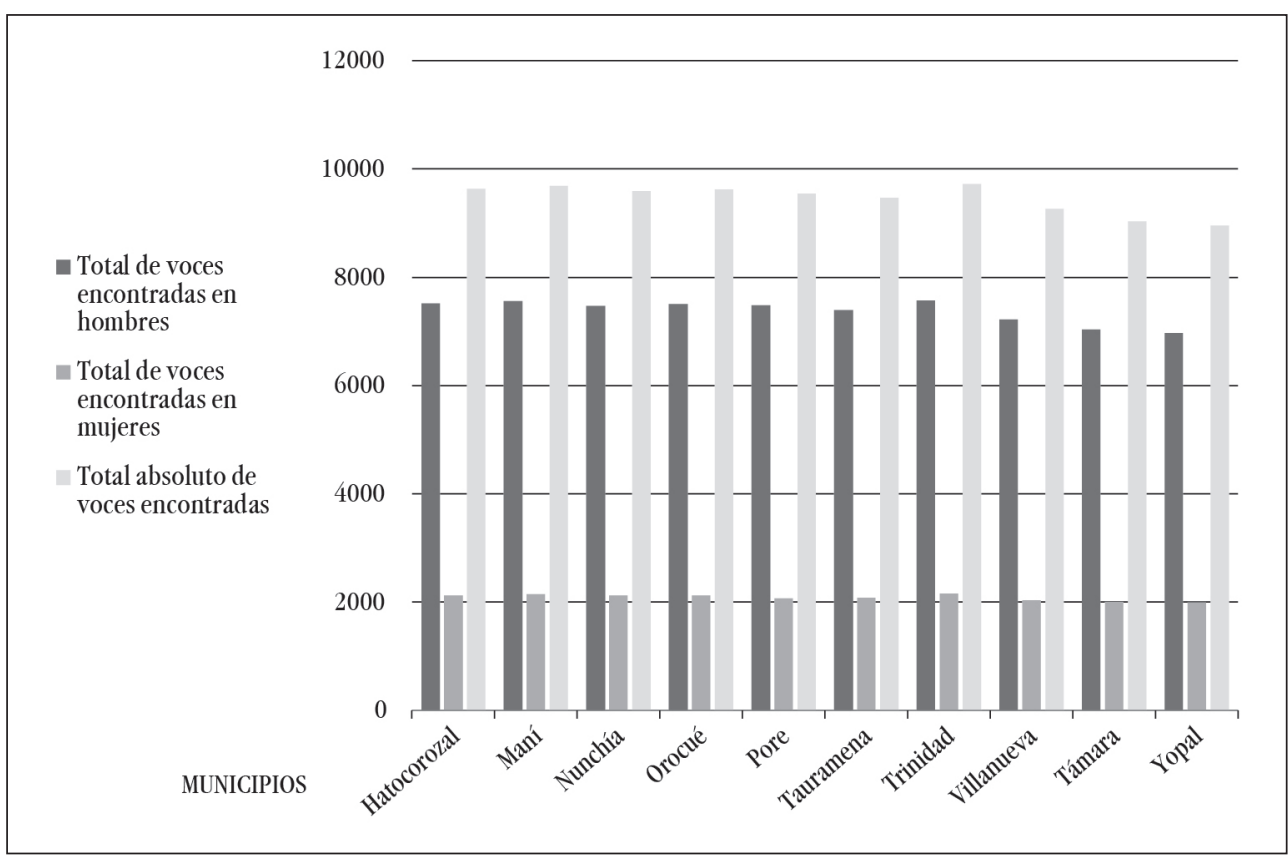

Figura 1. El dialecto. Ubicación y relaciones 
En esta figura, se totaliza por municipio el número de voces encontradas, tanto en el género masculino como femenino y en el total de las 90 encuestas llevadas a cabo en los municipios seleccionados. Aunque las voces se repiten en cada uno de los encuestados, en todos los municipios seleccionados, el mayor número de voces se encontró en Maní, esto es, 7554 voces en hombres y 2139 en mujeres.

Tabla 4. Comparativo entre léxico recogido, lengua estándar y hallazgos dialectales

\begin{tabular}{cc}
\hline TOTAL LÉXICO RECOGIDO & 1122 \\
\hline LENGUA ESTÁNDAR & 700 \\
\hline HALLAZGOS DIALECTALES & 422 \\
\hline
\end{tabular}

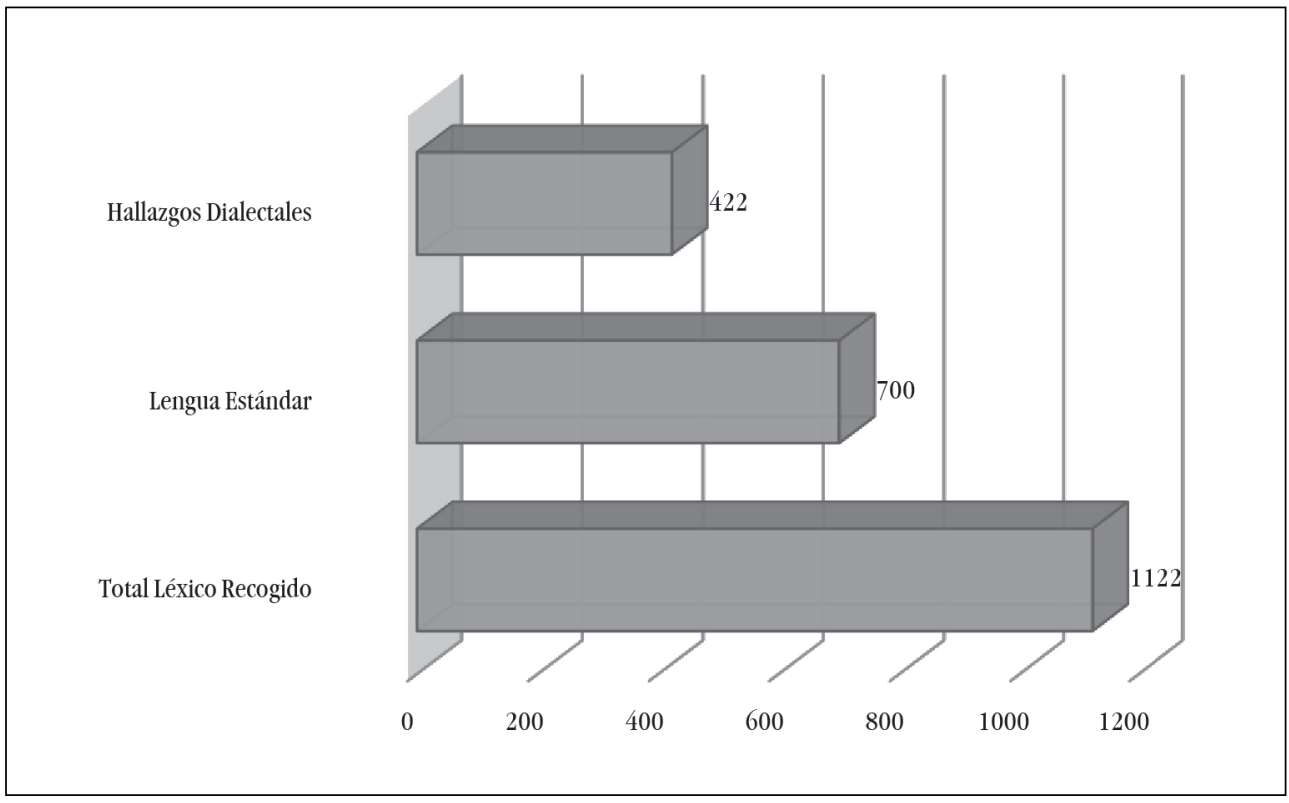

Figura 2. Gráfica comparativa entre léxico recogido, lengua estándar y hallazgos dialectales

La figura anterior nos permite observar que un número muy amplio de piezas léxicas de la ganadería en Casanare, corresponde a términos y expresiones ya existentes en la lengua estándar. El otro caso que se presenta en esta gráfica es la resemantización, es decir, la ampliación del significado. La palabra adquiere uno o varios significados diferentes a los que posee en la lengua estándar. Este procedimiento es muy generalizado en las acepciones encontradas. De las 1.122 palabras recogidas, 700 corresponden a la lengua estándar y 422 términos son considerados como dialecto regional. 
Tabla 5. Comparativo lengua estándar, diccionarios, hidroléxico, toponimias

\begin{tabular}{cccccc}
\hline LENGUA ESTÁNDAR & $\mathbf{7 0 0}$ & & & & \\
\hline DICCIONARIOS & $\begin{array}{c}\text { LENGUA } \\
\text { ESPAÑOLA }\end{array}$ & $\begin{array}{c}\text { AMERICA- } \\
\text { NISMOS }\end{array}$ & $\begin{array}{c}\text { COLOMBIA- } \\
\text { NISMOS }\end{array}$ & $\begin{array}{c}\text { SIKUANI- } \\
\text { ESPAÑOL }\end{array}$ & $\begin{array}{c}\text { ACHAGUA- } \\
\text { ESPAÑOL }\end{array}$ \\
\hline HIDROLÉXICO & 20 & 15 & 4 & 36 & 56 \\
\hline TOPONIMIAS & 36 & 24 & 5 & 27 & 43 \\
\hline
\end{tabular}

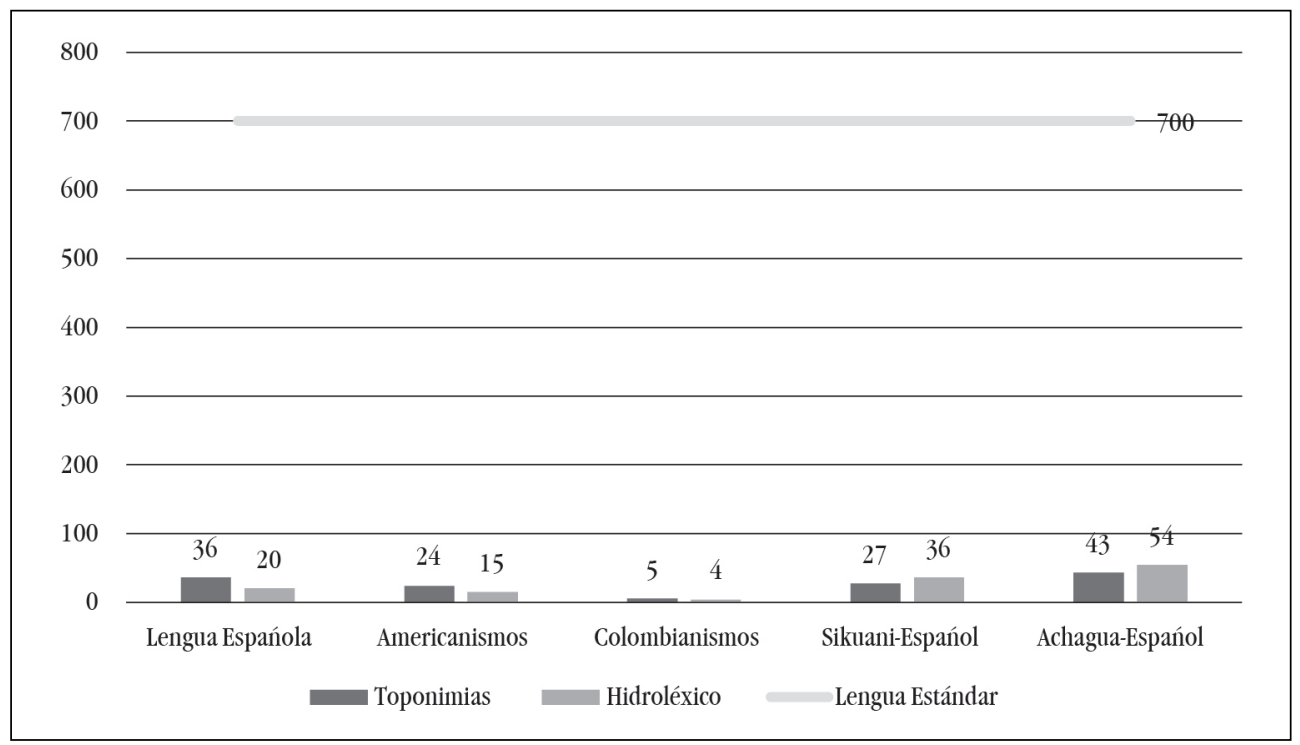

Figura 3. Comparativo lengua estándar, diccionarios, hidroléxico, toponimias

En la tabla y figura anteriores, se resalta que varias palabras presentes en la lengua estándar, pero que corresponden al hidroléxico y la toponimia llaneros, están presentes en los cinco diccionarios. Algunas están en el Diccionario de la lengua española (2012), otras en los demás: Diccionario de americanismos (2010); Breve diccionario de colombianismos (2012); Diccionario sikuani-español (Quixalós, 1988); Diccionario achagua-español/español-achagua (Meléndez Lozano, 2011).

\subsection{Análisis cualitativo}

En la investigación sobre el léxico dialectal de la ganadería en Casanare, se recogieron 1.122 palabras en los municipios de Hatocorozal, Maní, Nunchía, Pore, Orocué, Támara, 
Tauramena, Trinidad, Villanueva y Yopal. Una vez realizada la verificación de registro de cada unidad léxica en los diccionarios: Diccionario de la lengua española (Vigésima segunda edición, 2012), Diccionario de americanismos (2010), el Breve diccionario de colombianismos (4ª edición revisada, 2012), Diccionario sikuani-español (Primera edición), y el Diccionario achagua-español/español-achagua, (edición preliminar, 2011), se seleccionaron los términos que, aunque se encontraran en uno de ellos tuvieran significado diferente al encontrado en la región, como también las palabras que no aparecen en ninguno de los diccionarios consultados. Con ellos se elaboró el diccionario dialectal de la ganadería en Casanare.

Dentro del léxico de la ganadería en Casanare, se encuentra un dialecto social, ampliamente difundido en el área rural. Surgió y se desarrolla en la comunidad campesina de la parte plana del departamento, pero es utilizado parcialmente por sectores bajos, medios y altos, que encuentran en este léxico dialectal su identidad llanera, y las posibilidades de expresar de manera diferente sus vivencias. En la medida en que los medios masivos de comunicación han llegado a los habitantes casanareños, se acrecienta el desuso y con ello la pérdida de las expresiones dialectales por parte de los hablantes. El uso de los términos dialectales de la ganadería en Casanare se convirtió en un recurso retórico, que cumple funciones pragmáticas de acercamiento del emisor a sus interlocutores.

Es también muy representativo el número de 400 palabras que proceden de la lengua estándar, pero que adquieren un nuevo significado. Es por ello, que dichas piezas léxicas registradas en el corpus del léxico de la ganadería en Casanare se encuentran en el DLE.

Del corpus recolectado, 422 términos son considerados como hallazgos dialectales que enriquecerán el léxico de colombianismos, lo cual abre la posibilidad de desarrollar una investigación más amplia sobre el léxico de los llaneros casanareños. Esto no significa que ese sea el total de piezas léxicas de esta variedad dialectal, porque es un corpus abierto, en constante movimiento y cambio. Con frecuencia se incorporan nuevas palabras, se crean o se transforman otras, pero también algunas caen en desuso.

Al tratarse de una variedad dialectal antigua, en proceso de extinción y en constante cambio, no es posible hacer afirmaciones categóricas. Más bien se debe continuar con las observaciones, mantener el contacto con los informantes y seguir con el registro y la confrontación de los datos y el estudio a la luz de la teoría lingüística. Las palabras compuestas son frecuentes y no se salen de los modelos establecidos por el uso de la lengua estándar. Las variantes, la sinonimia y la polisemia son una característica común a muchas de las piezas léxicas que presentan varias alternativas para referirse a lo mismo. Cuanto más cotidianas sean las palabras, mayor es el número de significados o acepciones 
que presentan. Son casos de ensanchamiento semántico, propios de la lengua coloquial, en general. Los adjetivos son muy abundantes y presentan no solamente alta frecuencia de uso, sino también una carga semántica muy fuerte.

Del corpus analizado, 360 palabras son consideradas léxico dialectal y fueron organizadas alfabéticamente. Cada entrada tiene categoría gramatical y enseguida el(los) sentido(s) o significado(s). Aunque muchas forman parte del español general, las fuentes de referencia y consulta permitieron ratificar la necesidad de su registro, ya que el significado era distinto, lo cual ameritaba incluirlas.

Para tener un breve marco de referencia de las voces encontradas, se presentan las siguientes:

amortajao $\mathrm{m}$. Mezcla de la asadura de la res que se prepara a fuego lento, ensartándola en un chuzo de cubarro o de madera sazonada con sal.

claro m. Caldo elaborado con sangre de res, sal y cilantrón.

corinche $\mathrm{m}$. Ayudante de cocina. Mandadero.

hormiguillo $\mathrm{m}$. Enfermedad del casco de los equinos.

achicador $m$. Rejo corto para amarrar un animal a un botalón.

velador $m$. Persona que cuida el ganado para que no se produzca una estampida.

zaguero $\mathrm{m}$. Arreador de ganado que va a los lados del lote.

achicar v. Atar un animal pegado a un palo. Sacar el agua de la canoa.

cagaleriar v. Llevar la soga en el caballo cuando se enlaza trabajando toros.

Repartir la soga con cadeneta para fácil manejo.

güesiar v. Colear de a pie, luego de bajarse del caballo.

\section{Conclusiones}

Con esta investigación sobre el léxico de la ganadería en Casanare, variedad dialectal del español llanero en Colombia, a la que no se había prestado mucha atención desde la elaboración del $A L E C$, hasta hoy, se demuestra la existencia de un léxico dialectal de la ganadería en Casanare, que irrumpe con fuerza en una comunidad de hablantes y se manifiesta en la oralidad y casi nada en la escritura. 
Este léxico dialectal ha dejado su impronta no solo en los estratos sociales de Casanare, en los que surgió y se desarrolla, sino en toda la sociedad casanareña y colombiana. Por ello, puede afirmarse que estamos ante una variedad lingüística que merece no solamente este estudio, concentrado en el léxico dialectal, con el fin preparar el material para un diccionario, sino otras investigaciones lingüísticas que lo aborden desde diferentes perspectivas, como, por ejemplo, las particularidades sintácticas y fonéticas, que también lo diferencian de la lengua estándar; de igual manera, es importante llevar a cabo estudios semánticos y pragmáticos, que permitan una visión global de este fenómeno.

En fin, este estudio sobre el léxico dialectal de una variedad lingüística surgida en Casanare, Colombia, incursiona suministrando datos, reflexiones, y aportes útiles para posteriores trabajos.

\section{Referencias bibliográficas}

Academia colombiana de la lengua. (2012). Breve diccionario de colombianismos. Bogotá.

Alvar, M. (1996). Dialectología hispánica. Manual de dialectología hispánica. Barcelona: Ariel.

Alvar, M. (2000). En el Tesoro Léxico de las Hablas Andaluzas TLHA. Barcelona.

Cáceres, E., Salamanca, G., \& Mariño, L.M. (1988). Habla y cultura popular en los llanos de Casanare. Bogotá: Universidad de la Sabana.

Calderón Noguera, D.F., \& Alvarado Castellanos, J. (2011). El papel de la entrevista en la investigación sociolinguística. Cuadernos de Lingüística Hispánica, (17), 11-24. Recuperado de http:// revistas.uptc.edu.co/revistas/index.php/linguistica_hispanica/article/view/423

Cámara de Comercio de Casanare. (2013). Agenda de competitividad. Yopal: Cámara de Comercio de Casanare.

Dane. (2005). Bogotá.

Fabo, P. (1911). Idiomas y etnografía de la Región Oriental de Colombia. Barcelona, España: José Benet, Impresor.

Fajardo, A. (1993). La lexicografía del español en América. Madrid.

Heilmann, L. (1964). Por una dialectología estructural. Bologna.

Haensch, G., Wolf, L., Ettinger, S., \& Werner, R. (2012). La lexicografía: de la lingüística teórica a la lexicografía práctica. Madrid: Gredos. 
Hernández, R. (1998). Metodología de la investigación (2a ed.). México: McGraw-Hill.

Hernández, J. M., \& Trudgill, P. (2007). Diccionario de sociolingüística. Madrid: Gredos.

Hernández, R. et al. (2008). Metodología de la investigación (4 ed.). México: McGraw-Hill.

Instituto Caro y Cuervo. (1981). Atlas Lingüístico y Etnográfico de Colombia. Departamento de dialectología del Instituto Caro y Cuervo (Tomo II). Bogotá: Instituto Caro y Cuervo.

Instituto Caro y Cuervo. (2004). Caracterización léxica de los dialectos del español de Colombia según el Alec. Bogotá: Instituto Caro y Cuervo.

Lewandowski, T. (2000). Diccionario de lingüística (5 ed.). Madrid: Cátedra.

Martín, M. A. (1979). Del folclor llanero. Bogotá: Presencia.

Medina, G. (2003). Lexicografía española. Madrid: Ariel.

Meléndez, M. A. (2011). Diccionario achagua-español/español-achagua. Bogotá: Universidad de los Andes.

Montes, J. J. (1995). Dialectología general e hispanoamericana (3 ed.). Bogotá: Instituto Caro y Cuervo.

Porto, D. (2002). Manual de técnica lexicográfica. Madrid.

Queixalós, F. (1989). Diccionario sikuani-español (1 $1^{\mathrm{a}}$ ed.). Bogotá: Universidad de los Andes. Primera edición.

Rivera, S. (2001). Hombres con espolones de gallo ( $2^{\text {a }}$ ed.). Bogotá: Gente Nueva.

RAE. (2012). Diccionario de la lengua española. Madrid.

Reyes, y Córdoba. (2009). Los conceptos región y territorio como aporte a los estudios de la lengua. Bogotá: Instituto Caro y Cuervo.

Werner, R. (1993-1994). Cómo explicar el significado de las unidades léxicas en los diccionarios diferenciales. 